To cite this article:

Dejonckheere, E., Mestdagh, M., Verdonck, S., Lafit, G., Ceulemans, E., Bastian, B., \& Kalokerinos, E. K. (in press). The relation between positive and negative affect becomes more negative in response to personally relevant events. Emotion. https://doi.org/10.1037/emo0000697 


\title{
The Relation between Positive and Negative Affect becomes more Negative in Response to Personally Relevant Events
}

\author{
Egon Dejonckheere $^{1}$, Merijn Mestdagh ${ }^{1}$, Stijn Verdonck ${ }^{1}$, \\ Ginette Lafit ${ }^{1}$, Eva Ceulemans ${ }^{1}$, Brock Bastian ${ }^{2}, \&$ Elise Kalokerinos ${ }^{2}$ \\ ${ }^{1}$ KU Leuven - Faculty of Psychology and Educational Sciences \\ ${ }^{2}$ University of Melbourne - Melbourne School of Psychological Sciences
}

Correspondence concerning this article should be addressed to Egon Dejonckheere, Faculty of Psychology and Educational Sciences, KU Leuven, Tiensestraat 102, Leuven, 3000, Belgium. E-mail: egon.dejonckheere@kuleuven.be.

All supplemental data and code for this article can be accessed online at the Open Science Framework: https://osf.io/yte2w/. 


\begin{abstract}
Can we experience positive (PA) and negative affect (NA) separately (i.e., affective independence), or do these emotional states represent the mutually exclusive ends of a single bipolar continuum (i.e., affective bipolarity)? Building on previous emotion theories, we propose that the relation between PA and NA is not invariable, but rather fluctuates in response to changing situational demands. Specifically, we argue that our affective system shifts from relative independence to stronger bipolarity when we encounter events or situations that activate personally relevant concerns. We test this idea in an experience sampling study, in which we tracked the positive and negative emotional trajectories of 101 first-year university students who received their exam results, an event that potentially triggers a personally significant concern. Using multilevel piecewise regression, we show that running PA-NA correlations become increasingly more negative in the anticipation of results release, indicating stronger affective bipolarity, and ease back towards greater independence as time after this event passes. Furthermore, we show that this dynamic trajectory is particularly apparent for event-related PA and NA, and not affect in general, and that such shifts are partly a function of the importance people attribute to that event. We suggest that such flexible changes in the affect relation may function as an emotional compass by signalling personally relevant information, and create a motivational push to respond to these meaningful events in an appropriate manner.
\end{abstract}

Keywords: affective bipolarity, affective independence, goal-related emotion, experience sampling 


\section{The Relation between Positive and Negative Affect becomes more Negative in Response to Personally Relevant Events}

The question whether positive (PA) and negative affect (NA) are experienced independently (i.e., affective independence) or rather constitute the bipolar opposites of a single affective continuum (i.e., affective bipolarity) is not only a philosophical discussion (Diener, 1999; Tellegen, Watson, \& Clark, 1999), its answer also carries real-life implications (e.g., how one should tackle debilitating negative feelings; Russell \& Carroll, 1999). Where the original debate revolved around the nature of a fixed and universal affective structure, later theories abandoned this invariant view, investigating both between-person differences (e.g., Dejonckheere, Kalokerinos, Bastian, \& Kuppens, 2018; Feldman, 1995), as well as within-person changes in the affect relation (e.g., Reich, Zautra, \& Davis, 2003; Zautra, Berkhof, \& Nicolson, 2002). Here, building on this variable notion of the affect system, we aim to investigate the conditions under which the affect relation may shift from independence to bipolarity and vice versa.

\section{The Original Debate: A Fixed and Universal Affect Relation}

Early affect theories agreed on an affective structure that was generic and invariant, but differed in their view of whether PA and NA represent two separate unipolar dimensions or mutually exclusive opposites. Of the theories that hypothesize an independent relation between PA and NA, Watson and Tellegen (1985) may be considered most influential. In this model, PA and NA operate as two independent dimensions, with changes in PA providing us with little information about changes in NA (and vice versa). These researchers argue that an independent PA-NA relation is supported by evidence that both dimensions have distinct etiologies and operate through different bio-behavioral mechanisms (e.g., Watson, Wiese, Vaidya, \& Tellegen, 1999). 
Of the theories that hypothesize a bipolar relation between PA and NA, Russell's circumplex model of affect (1980) has received most attention. In this model, PA and NA represent the mutually exclusive ends of a single bipolar valence dimension that captures the hedonic quality of an emotion. As such, increases in PA are thought to go hand in hand with decreases in NA (and vice versa). Russell and colleagues find evidence for a bipolar PA-NA relation in different cultures (Russell, 1983), age groups, and emotional components (e.g., subjective experience versus facial expressions; Russell \& Bullock, 1985).

Although these two perspectives appear incompatible, later research reconciled these viewpoints by demonstrating that the extent to which PA and NA are independent versus bipolar opposites is dependent on a multitude of factors (Watson, 1988). For example, the strength of the PA-NA relation is known to be a function of the items that are used to construct PA and NA (Yik, Russell, \& Barrett, 1999), the time frame in which both affective states are assessed (Diener \& Emmons, 1984), response type formats (Russell \& Carroll, 1999), the inclusion of measurement error (Green, Goldman, \& Salovey, 1993), and the level of analysis (Brose, Voelkle, Lövdén, Lindenberger, \& Schmiedek, 2015)ํ․

With the recent expansion of mobile technology, researchers are able to track emotions in daily life using the experience sampling method (ESM; Csikszentmihalyi \& Larson, 2014). This within-person, longitudinal data collection method allows affective scientists to take on a more variable perspective on the affect relation, examining the role of both individual and situational factors. The investigation of both between-person differences and within-person changes in the affect relation may further add nuance to this seemingly timeless debate.

\section{Within-Person Changes in the Affect Relation}

\footnotetext{
${ }^{1}$ For more comprehensive reviews of the different positions regarding this debate, as well as in-depth discussions of the critical variables that determine the strength of the PA-NA relation see Bleidorn and Peters (2010), Dejonckheere et al. (2018), and Larsen, McGraw and Cacioppo (2001).
} 
Based on an integration of two prominent emotion theories, we propose that fluctuations in the PA-NA relation are context-dependent, driven by the experience of events or stimuli that activate personally relevant concerns. First, in their Control-Process View (CPV) on the origin and function of emotion Carver and Scheier (1990a) argue that affect stems from a self-regulatory process in which people constantly monitor their present actions (e.g., spending time with my wife), and compare these with an implicit internal standard (e.g., being a good husband). As life unfolds, we continuously make adjustments to our behavior, aiming to render any discrepancies with the proposed reference value minimal (Carver, 1979). Affect is thought to echo the degree with which this discrepancy reduction is succeeding (Carver \& Scheier, 1990b), with PA signalling accomplishment and fostering ongoing engagement with similar behavior, while NA indicates failure at meeting a particular standard and stimulates withdrawal or re-evaluation (Frijda, 1988). Thus, in relation to a single goal, the CPV theorizes that affect should be strictly bipolar (Carver \& Scheier, 1990a).

Yet, people typically pursue multiple goals in life, achieving their desired discrepancy reduction in some goal-related domains, but not in others. A consequence of this multitude of goals is that PA and NA are likely to be independent in everyday life (Carver \& Scheier, 1990a). Although the CPV does not explicitly address within-person changes in the affect relation, one could argue that the extent to which PA and NA are processed separately or as mutually exclusive opposites may vary with the dynamic prioritization of particular goals in one's life. That is, at times where one specific goal becomes relatively more central in a person's life (e.g., being a good husband may be more important around Valentine's day, compared to other goals such as furthering a career or maintaining good physical health), the associated reference value may predominantly determine that person's emotional state, which will either be positively or negatively valenced (e.g., Linville, 1985). In contrast, at times 
where no particular goal is overly central, many reference values will determine one's emotional state to a similar degree, allowing PA and NA to be experienced largely in parallel.

A second theory, proposed by Zautra and colleagues (2002), is the Dynamic Model of Affect (DMA), and explicitly discusses within-person changes in the affect relation. This theory hypothesizes, and establishes empirically, that the degree of independence between PA and NA varies with the experience of stressful life events ${ }^{2}$. By default, people may benefit from processing PA and NA in parallel, as maintaining two separate registers for affective information provides us with a maximum amount of information about our environment (Reich et al., 2003). In this way, under peaceful and predictable circumstances, the independent nature of PA and NA allows people to take into account both the positive and negative aspects of everyday situations, which permits the most optimal response at any given moment (Davis, Zautra, \& Smith, 2004). In times of stress or uncertainty, however, processing PA and NA separately becomes cognitively challenging, and the need to process affective stimuli rapidly likely outweighs their thoughtful and deliberate evaluation (Davis et al., 2004). Consequently, as our attentional focus becomes narrowed to ensure adaptive coping with the stressor, our emotional judgments are thought to become simplified and undifferentiated, reflected in the compression of PA and NA onto a single affective dimension (Zautra, Reich, Davis, Potter, \& Nicolson, 2000).

In essence, both theories (implicitly or explicitly) suggest that the relation between PA and NA changes according to situational demands. That is, they consider the structure of our affective life to be determined by events or stimuli that activate a personally relevant concern. Here, a concern refers to a latent motivational state that is associated with the ongoing pursuit

\footnotetext{
2 The DMA conceptualizes stress rather broadly, going beyond purely physiological and high-arousal reactions in response to aversive stimuli (Dejonckheere et al., 2018). In a DMA context, a stressor could entail any stimulus that moves people away from their homeostatic set point (Zautra, Affleck, Tennen, Reich, \& Davis, 1995).
} 
of a particular goal (Klinger, 1996), that results in an increased sensitivity for cues related to that goal and a readiness to act appropriately (Klinger \& Cox, 2004). Thus, both (a dynamic version of) the CPV and the DMA are similar in that they hypothesize that the affect relation will be pushed towards stronger bipolarity when a single concern moves to the forefront of our experience, because, for example, an event or stressor in our environment triggers its activation $^{3}$. In response to that concern, PA and NA levels are hypothesized to shift in an opposite direction, which should introduce an affective structure that becomes more unidimensional (e.g., Dejonckheere et al., 2018). As such, following the activation of a personally relevant concern, the diametric change in positive and negative affect intensity is thought to be the pivotal mechanism that produces the PA-NA correlation to become more negative (e.g., Folkman, 1997).

\section{Assessing Within-Person Changes in the Affect Relation in Daily Life}

Examining how emotional experiences play out in everyday life provides researchers with assessments that are particularly high in ecological validity, unparalleled by more traditional experimental designs (Bolger, Davis, \& Rafaeli, 2003). Nevertheless, because people differ in the standards or goals they prioritize (e.g., Lang \& Carstensen, 2002), as well as in the events and situations they encounter on a daily basis (e.g., Kahneman, Krueger, Schkade, Schwarz, \& Stone, 2004), assessing how the affect relation changes with the activation of personally relevant concerns may be challenging to investigate in a naturalistic context. To properly test the idea that the emotional system will be pushed towards bipolarity when a particular concern becomes salient in our experience, we need an event that both (a) triggers a personally relevant concern in a large group of participants, and (b) is relatively predictable for researchers to design a study around.

\footnotetext{
${ }^{3}$ In this way, we consider the DMA to be a specific instantiation of the (dynamic) CPV, in which the activated concern involves dealing with a stressor, with the ultimate goal to preserve a person's survival or (psychological) well-being (e.g., Klinger \& Cox, 2004).
} 
One event that meets both these requirements is the release of exam results. This is particularly the case among Belgian first-year university students. Because all Belgian students are allowed to enroll in a university program with almost no restrictions, a strong selection arises after the first semester, with many students having to revise their academic career because they received poor grades (e.g., the average percentage of passed subjects in this sample was only $56 \%, S D=35 \%$ ). As a consequence, receiving first semester results is an impactful experience, and likely triggers a concern (e.g., desire to receive good grades) that relates to a central goal in the life of these students (e.g., pursuing an academic career).

\section{The Current Study}

In the present research, we investigate how people's affect relation changes in response to an event that activates a concern of high personal relevance. We hypothesize that at times where no single concern is of particular central relevance, PA and NA should be experienced more independently. In contrast, when a single concern becomes relatively more central, the relation between PA and NA should be pushed towards greater bipolarity.

To test this idea in daily life, we tracked the positive and negative emotional trajectories of 101 first-year university students in the period before and after they received their first-semester grades using ESM. This allowed us to assess changes in the affect relation in response to this impactful event. Our central hypothesis was that, in the anticipation of their exam results release, students' PA-NA relation would become increasingly more bipolar (i.e., a correlation becoming more negative), as this event boosted the salience of one particular concern. After results release, we predicted that the PA-NA relation would wane back to independence (i.e., a correlation becoming more positive), as this concern lost its central status in the students' daily life. Drawing from the theories we outlined earlier, we built on this central hypothesis in two additional ways.

\section{Event-Related Versus Global Affect}


We hypothesized that this shift between greater independence and stronger bipolarity would be more apparent for event-related emotions than for global affect. Because the CPV argues that global affect is determined by many reference values simultaneously (Carver \& Scheier, 1990a), people are thought to experience general positive and negative affect always as relatively independent. In contrast, affective states that are specifically elicited by a personally relevant event should become more mutually exclusive around the time the associated concern is most salient in people's mind.

\section{Event Importance}

Because the CPV suggests that positive and negative affect are predominantly determined by reference values people deem important (Carver \& Scheier, 1990b), we evaluated whether the magnitude of change in the PA-NA relation was a function how important students considered their exam results (e.g., Steiner, Trabasso, \& Liwag, 1993). We investigated this idea both in terms of within-person changes (i.e., via multiple momentary assessments), as well as in terms of between-person differences (i.e., via a single trait-level assessment). From a within-person perspective, we hypothesized that momentary changes in the (exam-related) PA-NA relation should align with changes in the attributed importance to that event. That is, at times where students consider their exam results more important, their PA-NA relation is expected to show stronger affective bipolarity. Second, at the betweenperson level, we hypothesized that a push towards stronger affective bipolarity should be particularly evident in students who consider their exam results more important. In addition, moving back to affective independence should take longer for students who considered their exam grades more important.

\section{Method}

The current study was part of a larger project investigating the relation between different aspects of emotion, context, and psychological well-being (Kalokerinos, Erbas, 
Ceulemans, \& Kuppens, 2019; Study 2), and was ethically approved by the KU Leuven institutional review board.

\section{Participants}

We enrolled 101 first-year KU Leuven psychology students who had taken their first semester exams, and who would receive their grades in the near future ( 87 women; $M_{\text {age }}=$ 18.64; $\left.S D_{\text {age }}=1.45\right)$. This sample size allowed us to detect small to medium effects at the between-person level $(r=.30, \alpha=.05)$. We advertised our study at a first-year research participation session and via social media. Participants could receive up to $€ 50$ for their participation, with their actual compensation depending on their compliance with the ESM protocol (i.e., a decrease of $€ 5$ per $10 \%$ less than $80 \%$ of the total beeps completed). All participants provided informed consent.

\section{Procedure and Materials}

Three days before their exam results would be released, participants came to the lab in small groups and were instructed on the ESM protocol. We explained to participants that the study was about emotions and exams, but they were blind to any specific hypotheses. We did explicitly remind them, however, what day their results would be made available, so that they knew to anticipate the release. On the next day, participants started the ESM phase, meaning that the first two sampling days were pre-exam results. On the day of their results release (i.e., third day of the study), students were alerted via email that they could check their grades in an online portal, and we instructed them to do so immediately upon being notified. On this day, participants also received a link to an online questionnaire asking them to report their grade for each course, as well as to rate the importance they attributed to their results. Participants continued the ESM protocol until 6 days after results release. For a visual overview of the study protocol, including the key variables that were assessed in each phase, see Supplemental Materials 1. 
ESM protocol. We assessed changes in the within-person affect relation using experience sampling. Participants either installed the ESM application mobileQ (Meers, Dejonckheere, Kalokerinos, Rummens, \& Kuppens, in preparation; https://mobileq.org) on their own Android phone $(n=28)$, or were given a research-dedicated Motorola lab smartphone when their own device was incompatible with the software $(n=73)$. After providing participants with detailed information about the ESM protocol and survey (including a few practice trials in the presence of an experimenter), we instructed them to carry the phone everywhere they went.

The ESM protocol lasted for 9 consecutive days. Specifically, we prompted participants during the 2 days before the release of their exam results, on the day they received their grades, and for 6 days after results release. Each day, between 10 AM and 10 PM, we randomly beeped participants 10 times according to a stratified random interval scheme, which yielded a total of 90 beeps (ESM surveys) to be completed. On average, participants received a beep every 72 minutes $(S D=30 \mathrm{~min})$. As there was some variability in when students could check their grades, there were small differences in the number of preversus post-results surveys per participant, yet all participants received their results between beep 21 and $28(M=22 ; S D=1.25)$. Participants completed an average of $91 \%$ of the beeps $(S D=7.32 \%)$, reflecting good compliance with the protocol.

At each beep, participants completed a questionnaire about their momentary experience of various exam-related emotions, as well as their positive and negative affect in general. They also reported the momentary importance they attributed to their exam results. For all items, participants answered using a continuous slider, ranging from 0 (not at all) to 100 (very much). A complete overview of the ESM items we assessed (i.e., including the ones that were included to test other research questions, but are not used in this report), is available online at the Open Science Framework (https://osf.io/yte2w/). 
Exam-related PA and NA. Participants rated the intensity of four positive (happy, content, proud, relieved) and six negative (sad, stressed, anxious, angry, disappointed, ashamed) emotions in relation to their exam results (i.e., When you think about your grades right now, how ___ are you feeling?) in a random order. With the selection of these examrelated affect items we intended to assess emotional states that (a) are frequently experienced in a goal-related context (Tyson, Linnenbrink-Garcia, \& Hill, 2009; Pekrun, 2006), and (b) show considerable differences in valence and arousal (Moors et al., 2013), the two most prominent dimensions that underlie an affective experience (Feldman, 1995). To obtain scale scores for exam-related PA and NA, we averaged same-valenced emotion items at each beep ${ }^{4}$. We computed internal consistencies following Nezlek (2017), which showed excellent reliability at the person-level $\left(\alpha_{\mathrm{PA}}=.99 ; \alpha_{\mathrm{NA}}=.99\right)$, and good reliability at the beep-level $\left(\alpha_{\mathrm{PA}}\right.$ $\left.=.87 ; \alpha_{\mathrm{NA}}=.75\right)$.

Global PA and NA. In addition to the items that specifically assessed participants' emotional state towards their exam results, we also assessed how positive and negative they felt in the moment in general (i.e., not in relation to any particular event or stimulus). To minimize the number of questions at each beep, we assessed participants' momentary experience of global positive and negative affect with two single items (i.e., Please indicate how positive / negative you are feeling right now.).

To minimize the possibility that exam-specific information was over-weighted in participants' global affect evaluation, we always assessed global PA and NA first, followed by the random presentation of the different event-specific emotions. Furthermore, in the

\footnotetext{
${ }^{4}$ Because different exam-related PA and NA composites could yield differences in the strength of their mutual relation (e.g., Feldman \& Russell, 1998), we investigated the impact of our exam-related PA and NA operationalization on our findings in a multiverse analysis (Steegen, Tuerlinckx, Gelman, \& Vanpaemel, 2016). Specifically, we computed every unique exam-related PA-NA relation from the 4 positive and 6 negative emotion items $(n=945)$. Results can be found in Supplemental Materials 3 and show our conclusions are largely robust across different exam-related PA and NA operationalizations.
} 
baseline session prior to the ESM protocol, we explicitly emphasized that the first two items (i.e., global affect) concerned how they currently felt overall, while all subsequent questions were about their exam grades specifically (cfr. overview ESM items).

Momentary importance. To investigate whether within-person changes in students' affect relation coincided with changes in how important they considered their exam results, we assessed the momentary importance they attributed to their exam results via a single item (i.e., When you think about your grades right now, how important are your grades for you?).

Exam questionnaire. On the day of results release, participants were instructed to complete an additional questionnaire immediately after they had checked their grades. In this online survey, students both reported how important they considered the outcome of their exam results, as well as their grades for each subject. In total, five first-year psychology courses were offered, and most students took all five $(n=92)$.

General importance. To investigate whether individual differences in general importance attributed to students' exam grades were associated with differences in the dynamic trajectory of the affect relation, we assessed how important participants considered their exam results at the time of release via a single item (i.e., How important are these exam grades for you?). Students provided their answer on a Likert scale that ranged from 1 (not important at all) to 7 (very important). Overall, participants considered their exam results fairly important $(M=6.03 ; S D=1.15)$, illustrating the central relevance of this event in their lives ${ }^{5}$.

\section{Statistical Analyses}

\footnotetext{
${ }^{5}$ In addition to the general importance students attributed to their exam results, we investigated whether dynamic changes in students' affect relation were a function of their actual grades (operationalized as the percentage of subjects passed). In this way, we aimed to explore whether a responsive shift towards affective bipolarity was dependent on the actual outcome of this meaningful event (i.e., whether students received good results or not). Results can be found in Supplemental Materials 4 and illustrate that this was not the case.
} 
The data and R-code to reproduce all analyses reported in this article are available online at the Open Science Framework (https://osf.io/yte2w/).

Running correlations. Experience sampling yielded four separate affective time series per participant, exam-related PA and NA, and global PA and NA. Focal to our research question, we were interested in the dynamic trajectories of two different affect relations, participants' exam-related PA-NA relation and global PA-NA relation.

To see how a particular PA-NA relation changed over time, we calculated running correlations between two specific PA and NA states per individual (e.g., Cabrieto et al., 2018). To achieve this, we slid a window across both affective time series, moving one time point at a time (window size $=15$ time points) ${ }^{6}$. Per moving window, we calculated the Pearson correlation between two affective states, using only the time points within a particular window. Figure 1 illustrates how the exam-related PA-NA running correlation was obtained for a real participant in our dataset. The value of each running correlation was set at the midpoint of each corresponding window. We repeated this procedure until the end of the time series, but deleted running correlations that contained both pre- and post-results affective information, as this confound would create unreliable slopes in our subsequent piecewise regression analyses. Consequently, this introduced some missing values around the time participants checked their exam results (see Figure 1). On average, this moving window approach produced 62 running affect correlations per participant $(S D=3)$, which we deemed sufficient for assessing dynamic changes in people's affective structure based on previous studies (e.g., Folkman, 1997).

\footnotetext{
${ }^{6}$ We consider 15 time points to reflect an optimal window size. Larger window size allow more reliable running affect correlations, but result in fewer measurement occasions for the piecewise regression analyses. In contrast, smaller window sizes produce running affect correlations that are more prone to noise, but yield more measurement occasions for piecewise regression. Nevertheless, to check the robustness of our findings in terms of different window sizes, we repeated our piecewise regression analyses with different windows. Results can be found in Supplemental Materials 5 and fully support our conclusions reported here.
} 
[Figure 1 around here]

Multilevel piecewise regression. To account for the nested structure of the data (i.e., PA-NA running correlations within participants) we conducted our analyses in a multilevel framework (using lme4; Bates, Maechler, Bolker, \& Walker, 2015). Specifically, we used multilevel piecewise regression to compare whether changes in the affect relations differed pre- versus post-results release ${ }^{7}$

To this end, we first recoded the time variable (i.e., an ordinal beep identifier) for each participant, so that zero would reflect the first beep after students had checked their grades. This recoding facilitated the interpretation of our intercept, as it now indicated how PA and NA were related around results release (the moment where academic aspirations are likely most activated). Next, we introduced a dummy variable that indicated whether a particular affect running correlation was assessed before (0) or after (1) exam results were checked. Focal to our research question, we examined how this dummy variable interacted with the recoded time variable, as this interaction revealed how the trajectory of the affect running correlation differed before and after exam results were released.

Thus, in our first series of multilevel models, we predicted how a particular (examrelated or global) PA-NA running correlation was a function of an intercept, a time variable, and the interaction between the time and the dummy variable ${ }^{8}$. This interaction introduced the

\footnotetext{
${ }^{7}$ Because our moving window approach potentially violated the assumption that the values of our outcome variable were independently and identically distributed (e.g. Lütkepohl, 2005), we also fitted our multilevel piecewise regression models on non-overlapping windows, in order to remove any serial dependencies between consecutive PA-NA correlations. Although this resulted in substantially fewer PA-NA correlations per student (i.e., an average of 12 independent affect correlations per participant each based on \pm 8 unique data points), our results were highly comparable to the ones presented here (see Supplemental Materials 6). However, when differences between the two approaches occurred, we report this in the main text of this article.

${ }^{8}$ A main effect for the dummy variable was not included in our model for parsimony reasons. First, differences in the average affect relation pre versus post results release were not central to the current research question (i.e., the dynamic trajectory of students' PA-NA relation). Second, if included in the prediction of their event-related PA-NA relation, this main effect was non-significant $(\beta=.129, \mathrm{SE}=.09, t=1.47, p=.142)$.
} 
piecewise component to our models. The intercept and slopes of both predictors were allowed to vary across participants. This introduced the multilevel component to our models. The specific equations for these basic multilevel piecewise models can be found in Supplemental Materials 2.

In a next series of models, to investigate whether within-person changes in students' (exam-related or global) PA-NA correlation were a function of individual differences in the general importance attributed to their exam results, we included (a grand-mean centered version of) this variable at level 2 , and evaluated the cross-level interaction with each effect at level 1.

Finally, to evaluate the within-person association between students' momentary attributed exam importance and their affect relation, we tested a different multilevel model with a single predictor, (a within-person centered version of) students' momentary importance.

\section{Results}

[Table 1 around here]

\section{Event-Related Versus Global Affect}

The results for the multilevel piecewise regression models predicting changes in people's affect relations are summarized in Table 1. First, in the prediction of students' examrelated affect relation, we observed a significant and negative effect of time before grades were checked (see Figure 2). This implies that in the anticipation of their exam results, students' exam-related PA-NA relation became increasingly more bipolar, which is in line with our predictions ( $M$ exam-related affect relation around 48 hours before results release $=$ $.09, S D=.54$; around 24 hours before results release $=-.18, S D=.41)$. Central to our research question, this affect relation waned back to greater independence after students had checked their grades, which was reflected in a significant and positive effect of time after exam results 
were released, as well as a significant interaction between the pre-results and post-results time slope $(M$ exam-related affect relation around 24 hours after results release $=-.21, S D=.42$; around 48 hours after results release $=-.11, S D=.42$; around 72 hours after results release $=-$ $.05, S D=.42)^{9}$.

[Figure 2 around here]

In contrast to this finding, but conforming with our hypothesis, students' global affect relation did not follow such a trajectory. The relation between students' global momentary PA and NA did not become increasingly more negative in the anticipation of their exam results, nor did we observe a significant interaction between the effect of time before versus after results were checked. We did observe, however, a significant and positive effect of time after grades were released, indicating that students' global PA-NA relation became more independent as time after this event passed ${ }^{10}$.

\section{Event Importance}

Next, we investigated whether dynamic shifts in the affect relation were a function of the importance students attributed to their exam results. Table 2 presents both the results for the multilevel models that tested the within- and between-person effects of students' attributed importance on their changing affect relations.

[Table 2 around here]

\footnotetext{
${ }^{9}$ We also wanted to rule out that this dynamic trajectory was a statistical artefact, driven by the restricted variance in exam-related PA and / or NA as a result of an increasing floor (for exam-related NA) and / or ceiling effect (for exam-related PA) towards the end of the ESM protocol. To this end, we reran the original models, but additionally controlled for local differences in affect intensity of, and variability in, exam-related PA and NA. Results are presented in Supplemental Materials 7, and show this dynamic trajectory of participants' exam-related affect correlation remains evident after taking into account the effect of these covariates. Thus, there is no evidence that our findings are the result of floor and / or ceiling effects.

${ }^{10}$ However, a post-hoc paired samples $t$-test in which we mutually compared the strength of both students' personspecific post-results slopes revealed that the effect of time on their global PA-NA correlation was significantly weaker than on their event-related PA-NA correlation, $t(100)=2.31, p=.023$.
} 
For the within-person model, we observed a negative association between the examrelated PA-NA relation and momentary importance. That is, at times where students considered their exam results more important, the relation between their positive and negative exam-related affect was more bipolar. In contrast, for the global PA-NA relation, we did not observe a within-person effect of momentary results importance.

For the between-person model, in the prediction of the exam-related PA-NA relation, we found that the effect of time before results release was significantly stronger for people who generally attributed more importance to their exam results. That is, the rate with which their exam-related affect relation became increasingly more bipolar in the anticipation of their exam grades was faster (this effect became non-significant when non-overlapping windows were used; see Supplemental Materials 6). At the moment of release, however, we did not observe stronger affective bipolarity for students who generally attributed more importance to their exam results, as there was no significant cross-level interaction between the intercept and students' attributed importance. Similarly, the attributed importance to their exam results had no significant effect on the speed with which the exam-related PA-NA correlation moved towards stronger independence. The three-way cross-level interaction, however, was significant, which indicates that the interaction between the effect of time before versus after results were checked, was stronger for students who generally attributed more importance to their exam results (this effect became non-significant when non-overlapping windows were used; see Supplemental Materials 6). In sum, these findings only partially support our hypotheses.

Finally, in line with our predictions, for students' global PA-NA relation, the general attributed importance attributed to their exam results never impacted the within-person dynamics of this affect relation.

\section{Discussion}


With this research, we advanced a variable conception of the affect system, aiming to investigate how people's relation between positive and negative affect may oscillate between relative independence and stronger bipolarity. Unifying two influential emotion theories, we proposed that when events or stimuli that activate personally relevant concerns occur, the affect relation shifts adaptively from affective independence to stronger bipolarity. In contrast, at times where no single concern is of central relevance, positive and negative affect are experienced more in parallel.

\section{Event-Related Versus Global Affect}

Investigating this idea in an experience sampling study, we found that students' positive and negative emotions related to their exam grades became increasingly more mutually exclusive as the moment of their results release approached, and receded towards a more independent experience as time after this event passed. Taken together with previous work that established the dynamic nature of this relation (e.g., Folkman, 1997, Zautra et al., 2002), this variable quality refutes earlier theories on the structure of affect that conceived the affect relation to be fixed (e.g., Russell, 1980; Watson \& Tellegen, 1985).

Because the publication of their exam results likely heightened the relative salience of students' academic aspirations, we posit that this event induced a temporary reprioritization of the concerns in students' mind. Hence, as this particular concern moved to the forefront of their experience, this was associated with a more bipolar experience of positive and negative exam-related affect because these affective states shifted in an opposite direction (e.g., Carver \& Scheier, 1990b; Folkman, 1997; Dejonckheere et al., 2018). Notably, a responsive shift towards stronger affective bipolarity was evident independent of the actual outcome of this meaningful event (i.e., whether students actually received good exam grades or not; see Supplemental Materials 4). This suggests that the activation of the associated concern in itself may be sufficient to push people's related PA and NA towards bipolarity, and that the 
dynamic trajectory of this PA-NA relation is not particularly driven by the (un)successful realization of that underlying goal (Carver \& Scheier, 1990a).

In contrast, we did not observe this dynamic pattern for students' global affect. Following the CPV (Carver \& Scheier, 1990a, 1990b), global PA and NA levels are thought to be driven by a concurrent interplay between many concerns that are of personal relevance, meaning that the mutual relation between general positive and negative affect is less susceptible to a single meaningful event. However, it is possible that in response to extreme events, even people's global affect relation may show a tendency towards stronger bipolarity (e.g., as reaction to the death of a spouse or child; Coifman, Bonanno, \& Rafaeli, 2007). Indeed, research shows that during episodes of very strong and intense positive or negative emotionality, the simultaneous experience of both affective states becomes incompatible (Diener \& Iran-Nejad, 1986).

\section{Event Importance}

Next, we found that how important students considered their exam results was related to the dynamic trajectory of their exam-related affect relation. Within individuals, moment-tomoment fluctuations in exam importance were associated with changes in students' examrelated PA-NA relation, with higher importance relating to stronger affective bipolarity. This finding supports the idea that the relative importance of a particular concern may play a role in the strength of people's event-related affect relation (e.g., Linville, 1985; Steiner et al., 1993). At times where a personal concern is particularly prominent, the associated affective states are experienced more as mutually exclusive opposites, with positive feelings in relation to that concern ruling out the experience of negative ones and vice versa.

Likewise, between individuals, there was some evidence that students who deemed their exam grades as more important showed a more pronounced dynamic pattern in their exam-related affect relation, although these results were more mixed. Specifically, in those 
who found their exam grades more important, the speed with which their positive and negative feelings became increasingly more mutually exclusive was faster in anticipation of their exam results. This speedy push, however, did not result in stronger affective bipolarity around the time results were released. Similarly, there was also no effect of between-person event importance on the rate with which affective life moved towards greater independence.

\section{Functionality of a Variable Affect Relation}

Although this idea remains somewhat speculative, there are reasons to believe that context-dependent fluctuations in the PA-NA relation may be adaptive (e.g., Dreisbach \& Fröber, 2019). That is, a flexible affective system that switches from independence to stronger bipolarity could function as an emotional compass that draws our focus to personally significant information, as it signals the activation of a concern we consider important. In line with the DMA (Zautra et al., 2000), a unidimensional emotional life that generates an affective experience that is either positive or negative directly communicates whether our personal interests and concerns are met or not. In turn, this emotional evaluation may instigate a motivational push that guides our thoughts and behavior to respond to that information in an appropriate way (e.g., Frijda, 1988; Klinger \& Cox, 2004).

\section{Limitations and Future Directions}

Although the current study provides promising initial evidence for the link between dynamics in the structure of affect and the activation of personally relevant concerns, the results should be considered in the light of some limitations, which we hope future studies will address.

First, there are constraints on generality regarding the stressor we investigated (Simons, Shoda, \& Lindsay, 2017). The stressor we studied (i.e., receiving exam grades) was a relatively clear-cut and isolated event, and it remains unclear to what extent the academic context in which we tested our hypotheses is representative of events or stimuli that activate 
different personally relevant concerns (e.g., a romantic break-up or job promotion). For example, as noted by Carver and Scheier (1990b), a more complex event may evoke multiple views on the personally relevant concern it elicits, which would attenuate the corresponding PA-NA relation (e.g., a blend of sadness and relief when you consider that a romantic breakup may also create new opportunities; McCarthy, Lambert, \& Brack, 1997). In a similar vein, a single event may sometimes elicit multiple personally relevant concerns that are not necessarily perfectly compatible (Carver \& Scheier, 1990b). The activation of multiple concerns could again give rise to a more complex, and hence less bipolar, emotional response (e.g., Berrios, Totterdell, \& Kellett, 2014). For example, a job promotion may satisfy the goal of furthering your career (generating PA), but may also inhibit other relevant concerns such as maintaining good social relationships because your friend and colleague did not get promoted (generating NA). In this respect, future research could benefit from moving beyond the study of a single concern, in order to reflect the myriad of more complex concerns we face in our everyday lives.

Second, it will be important for future research to directly evaluate the theoretical mechanism behind these effects: the underlying reprioritization of people's concerns. Although we assessed the moment-to-moment importance students attributed to their exam results, we can only implicitly infer that students' aspirations to academically achieve well are relatively maximized around the release of their exam results. Assessing the relative salience of particular concerns in a more explicit way (e.g., evaluating changes in relative goal programming; Aköz \& Petrovic, 2007) would advance our direct understanding of the processes that are involved in the dynamic fluctuations of the affect relation.

Finally, the comparison between the multi-item exam-related and single-item global affect was not ideal. Not only were the single-item measures for global affect likely less reliable, differences in emotional content also made an absolute comparison of affective 
bipolarity infeasible, because different PA and NA item assessments are known to differ in the strength of their mutual relation (e.g., Dejonckheere et al., 2018; Feldman \& Russell, 1998). As such, we were only able to compare relative within-person changes in people's event-related versus global PA-NA relation (i.e., differences in their respective dynamic trajectories), rather than making comparisons in the absolute magnitude of these two types of relations.

\section{Conclusion}

In sum, we found empirical support for a variable affect system that shifts from independence to stronger bipolarity when a personally relevant concern becomes more salient. This trajectory was apparent in event-related affect, but not affect in general, and was partly associated with the importance people attributed to that event. 


\section{Acknowledgments}

This research was supported by the research fund of KU Leuven (C14/19/054), by a Australian Research Council Discovery grant awarded to Brock Bastian (DP140103757), and by a grant of the Fund of Scientific Research Flanders (FWO) awarded to Eva Ceulemans (G074319N). The data collection was supported by a Marie Skłodowska-Curie fellowship (704298) awarded to Elise Kalokerinos. Elise Kalokerinos is supported by an Australian Research Council Discovery Early Career Researcher Award (DE180100352). Stijn Verdonck is supported by the Fund of Scientific Research Flanders (FWO). 


\section{References}

Link for all data and code on Open Science Framework: https://osf.io/yte2w/

Aköz, O., \& Dobrila, P. (2007). A fuzzy goal programming method with imprecise goal hierarchy. European Journal of Operational Research, 181, 1427-1433. https://doi.org/10.1016/j.ejor.2005.11.049

Berrios, R. Totterdell, P., \& Kellett, S. (2015). Investigating goal conflict as a source of mixed emotions. Cognition and Emotion, 29, 755-763.

https://doi.org/10.1080/02699931.2014.939948

Bates, D., Maechler, M., Bolker, B., \& Walker, S. (2015). Fitting linear mixed-effects models using lme4. Journal of Statistical Software, 67, 1-48. https://doi.org/10.18637/jss.v067.i01

Bleidorn, W., \& Peters A. L. (2011). A multilevel multitrait-multimethod analysis of self- and peer-reported daily affective experiences. European Journal of Personality, 25, 398408. https://doi.org/10.1002/per.804

Bolger, N., Davis, A., \& Rafaeli, E. (2003). Diary methods: Capturing life as it is lived. Annual Reviews in Psychology, 54, 579-616. https://doi.org/10.1146/annurev.psych.54.101601.145030

Brose, A., Voelkle, M. C., Lövdén, M., Lindenberger, U., \& Schmiedek, F. (2015). differences in the between-person and within-person structures of affect are a matter of degree. European Journal of Personality, 29, 55-71.

Cabrieto, J., Tuerlinckx, F., Kuppens, P., Wilhelm, F. H., Liedlgruber, M., \& Ceulemans, E. (2018). Capturing correlation changes by applying kernel change point detection on the running correlations. Information Sciences, 447, 117-139. https://doi.org/10.1016/j.ins.2018.03.010 
Carver, C. S., \& Scheier, M. F. (1990a). Principles of self-regulation: Action and emotion. In Handbook of motivation and cognition: Foundations of social behavior, Vol. 2. (pp. 3-52). New York, NY, US: Guilford Press.

Carver, C. S., \& Scheier, M. F. (1990b). Origins and functions of positive and negative affect: A control-process view. Psychological Review, 97, 19-35. https://doi.org/10.1037/0033-295X.97.1.19

Coifman, K. G., Bonanno, G. A., \& Rafaeli, E. (2007). Affect dynamics, bereavement and resilience to loss. Journal of Happiness Studies, 8, 371-391. http://dx.doi.org/10.1007/s10902-006-9014-5

Csikszentmihalyi, M., \& Larson, R. (2014). Validity and reliability of the experiencesampling method. In M. Csikszentmihalyi (Ed.), Flow and the foundations of positive psychology: The collected works of Mihaly Csikszentmihalyi (pp. 35-54). Dordrecht: Springer Netherlands. https://doi.org/10.1007/978-94-017-9088-8_3

Davis, M. C., Zautra, A. J., \& Smith, B. (2004). Chronic pain, stress, and the dynamics of affective differentiation. Journal of Personality, 72, 1133-1159. https://doi.org/10.1111/j.1467-6494.2004.00293.x

Dejonckheere, E., Kalokerinos, E., Bastian, B., \& Kuppens, P. (2018). Poor emotion regulation ability mediates the link between depressive symptoms and affective bipolarity. Cognition and Emotion, 33, 1076-1083. https://doi.org/10.1080/02699931.2018.1524747

Dejonckheere, E., Mestdagh, M., Houben, M., Erbas, Y., Pe, M., Koval, P., ... Kuppens, P. (2018). The bipolarity of affect and depressive symptoms. Journal of Personality and Social Psychology, 114, 323-341. https://doi.org/10.1037/pspp0000186

Dejonckheere, E., Mestdagh, M., Houben, M., Rutten, I., Sels, L., Kuppens, P., \& Tuerlinckx, F. (2019). Complex affect dynamics add limited information to the prediction of 
psychological well-being. Nature Human Behaviour, 3, 478-491.

https://doi.org/10.1038/s41562-019-0555-0

Diener, E. (1999). Introduction to the special section on the structure of emotion. Journal of Personality and Social Psychology, 76, 803-804.

Diener, E., \& Emmons, R. A. (1984). The independence of positive and negative affect. Journal of Personality and Social Psychology, 47, 1105-1117.

Diener, E., \& Iran-Nejad, A. (1986). The relationship in experience between various types of affect, Journal of Personality and Social Psychology, 50, 1031-1038.

Dreisbach, G., \& Fröber, K. (2019). On how to be flexible (or not): Modulation of the stability-flexibility balance. Current Directions in Psychological Science, 28, 3-9. https://doi.org/10.1177/0963721418800030

Feldman, L. A. (1995). Valence focus and arousal focus: Individual differences in the structure of affective experience. Journal of Personality and Social Psychology, 69, 153-166. https://doi.org/10.1037/0022-3514.69.1.153

Feldman, L. A., \& Russell, J. A. (1998). Independence and bipolarity in the structure of current affect. Journal of Personality and Social Psychology, 74, 967-984. https://doi.org/10.1037/0022-3514.74.4.967

Folkman, S. (1997). Positive psychological states and coping with severe stress. Social Sciences and Medicine, 45, 1207-1221.

Frijda, N. H. (1988). The laws of emotion. American Psychologist, 43, 349-358. https://doi.org/10.1037/0003-066X.43.5.349

Green, D. P., Goldman, S. L., \& Salovey, P. (1993). Measurement error masks bipolarity in affect ratings. Journal of Personality and Social Psychology, 64, 1029-1041. 
Kahneman, D., Krueger, A. B., Schkade, D. A., Schwarz, N., \& Stone, A. A. (2004). A survey method for characterizing daily life experience: The day reconstruction method. Science, 306, 1776-1780. https://doi.org/10.1126/science.1103572

Kalokerinos, E. K., Erbas, Y., Ceulemans, E., \& Kuppens, P. (2019). Differentiate to regulate: Low negative emotion differentiation is associated with ineffective use but not selection of emotion-regulation strategies. Psychological Science. Online First Publication. https://doi.org/10.1177/095679761983876

Klinger, E. (1996). Emotional influences on cognitive processing, with implications for theories of both. In P. M. Gollwitzer \& J. A. Bargh (Eds.). The psychology of action: Linking cognition and motivation to behavior (pp. 168-189). New York: Guilford Press.

Klinger, E., \& Cox, W. M. (2004). Motivation and the theory of current concerns. In W.M. Cox, \& E. Klinger (Eds.), Handbook of motivational counseling: Concepts, approaches, and assessment (pp. 3-27). Chichester: Wiley.

Lang, F. R., \& Carstensen, L. L. (2002). Time counts: Future time perspective, goals, and social relationships. Psychology and Aging, 17, 125-139. https://doi.org/10.1037//0882-7974.17.1.125

Larsen, J. T., McGraw, A. P., \& Cacioppo, J. T. (2001). Can people feel happy and sad at the same time? Journal of Personality and Social Psychology, 81, 684-696. http://dx.doi.org/10.1037/0022-3514.81.4.684

Linville, P. W. (1985). Self-Complexity and Affective Extremity: Don't Put All of Your Eggs in One Cognitive Basket. Social Cognition, 3, 94-120. https://doi.org/10.1521/soco.1985.3.1.94

Lütkepohl, H. (2005). New Introduction to multiple time series analysis. Springer Science \& Business Media. 
McCarthy, C. J., Lambert, R. G., \& Brack, G. (1997). Structural model of coping, appraisals, and emotions after relationship breakup. Journal of Counselling and Development, 76, 53-64. http://dx.doi.org/10.1002/j.1556-6676.1997.tb02376.x

Meers, K., Dejonckheere, E., Kalokerinos, E., Rummens, K., \& Kuppens, P. MobileQ: An open-source experience sampling smartphone application., in preparation.

Moors, A., De Houwer, J., Hermans, D., Wanmaker, S., van Schie, K., Van Harmelen, A.-L., ... Brysbaert, M. (2013). Norms of valence, arousal, dominance, and age of acquisition for 4,300 Dutch words. Behavior Research Methods, 45, 169-177. https://doi.org/10.3758/s13428-012-0243-8

Nezlek, J. B. (2017). A practical guide to understanding reliability in studies of within-person variability. Journal of Research in Personality, 69, 149-155. https://doi.org/10.1016/j.jrp.2016.06.020

Pekrun, R. (2006). The control-value theory of achievement emotions: Assumptions, corollaries, and implications for educational research and practice. Educational Psychology Review, 18, 315-341. https://doi.org/10.1007/s10648-006-9029-9

Reich, J. W., Zautra, A. J., \& Davis, M. (2003). Dimensions of affect relationships: Models and their integrative implications. Review of General Psychology, 7, 66-83. https://doi.org/10.1037/1089-2680.7.1.66

Russell, J. A. (1980). A circumplex model of affect. Journal of Personality and Social Psychology, 39, 1161-1178. http://dx.doi.org/10.1037/h0077714

Russell, J. A. (1983). Pancultural aspects of the human conceptual organization of emotions. Journal of Personality and Social Psychology, 45, 1281-1288.

Russell, J. A., \& Bullock, M. (1985). Multidimensional scaling of emotional facial expressions: Similarity from preschoolers to adults. Journal of Personality and Social Psychology, 48, 1290-1298. 
Russell, J. A., \& Carroll, J. M. (1999). On the bipolarity of positive and negative affect. Psychological Bulletin, 125, 3-30.

Simons, D. J., Shoda, Y., \& Lindsay, D. S. (2017). Constraints on Generality (COG): A proposed addition to all empirical papers. Perspectives on Psychological Science, 12, $1123-1128$.

Steiner, N. L., Trabasso, T., \& Liwag, M. (1993). The representation and organization of emotional experience. Unfolding the emotional episode. In M. Lewis \& J. Haviland (eds.) Handbook of Emotion (pp. 279-299). Guilford, New York.

Steegen, S., Tuerlinckx, F., Gelman, A., \& Vanpaemel, W. (2016). Increasing Transparency Through a Multiverse Analysis. Perspectives on Psychological Science, 11, 702-712. https://doi.org/10.1177/1745691616658637

Tellegen, A., Watson, D., \& Clark, L. A. (1999). On the dimensional and hierarchical structure of affect. Psychological Science, 10, 297-303. https://doi.org/10.1111/14679280.00157

Tyson, D. F., Linnenbrink-Garcia, L., \& Hill, N. E. (2009). Regulating debilitating emotions in the context of performance: Achievement goal orientations, achievement-elicited emotions, and socialization contexts. Human Development, 52, 329-356. https://doi.org/10.1159/000242348

Watson, D. (1988). The vicissitudes of mood measurement: Effects of varying descriptors, time frames, and response formats on measures of positive and negative affect. Journal of Personality and Social Psychology, 55, 128-141. http://dx.doi.org/10.1037/0022-3514.55.1.128

Watson, D., Clark, L. A., \& Tellegen, A. (1988). Development and validation of brief measures of positive and negative affect: the PANAS scales. Journal of Personality and Social Psychology, 54(6), 1063-1070. 
Watson, D., \& Tellegen, A. (1985). Toward a consensual structure of mood. Psychological Bulletin, 98, 219-235. http://dx.doi.org/10.1037/0033-2909.98.2.219

Watson, D., Wiese, D., Vaidya, J., \& Tellegen, A. (1999). The two general activation systems of affect: Structural findings, evolutionary considerations, and psychobiological evidence. Journal of Personality and Social Psychology, 76, 820-838. http://dx.doi.org/10.1037/0022-3514.76.5.820

Yik, M. S. M., Russell, J. A., \& Barrett, L. F. (1999). Structure of self-reported current affect: Integration and beyond. Journal of Personality and Social Psychology, 77, 600-619. Zautra, A. J., Affleck, G. G., Tennen, H., Reich, J. W., \& Davis, M. C. (2005). Dynamic approaches to emotions and stress in everyday life: Bolger and Zuckerman reloaded with positive as well as negative affects. Journal of Personality, 73, 1511-1538.

Zautra, A. J., Berkhof, J., \& Nicolson, N. A. (2002). Changes in affect interrelations as a function of stressful events. Cognition and Emotion, 16, 309-318. https://doi.org/10.1080/02699930143000257

Zautra, A. J., Reich, J. W., Davis, M. C., Potter, P. T., \& Nicolson, N. A. (2000). The role of stressful events in the relationship between positive and negative affects: Evidence from field and experimental studies. Journal of Personality, 68, 927-951. 
Tables

Table 1. Assessing the dynamic trajectory of students' exam-related and global affect relation via multilevel piecewise regression.

\begin{tabular}{lccccccccccc}
\hline & \multicolumn{4}{c}{ Exam-related PA-NA correlation } & \multicolumn{3}{c}{ Global PA-NA correlation } \\
\cline { 2 - 9 } Predictors & $\beta$ & $\mathrm{SE}$ & $t$ & $p$ & $95 \% \mathrm{CI}$ & $\beta$ & $\mathrm{SE}$ & $t$ & $p$ & $95 \% \mathrm{CI}$ \\
\hline Intercept & -.244 & .04 & -5.96 & $<.001$ & $-.32--.16$ & -.358 & .04 & -8.55 & $<.001$ & $-.44--.28$ \\
Slope time pre-results & -.012 & .004 & -2.68 & .007 & $-.02--.003$ & .004 & .004 & 0.88 & .378 & $-.005-.01$ \\
Slope time post-results $*$ & .005 & .001 & 5.39 & $<.001$ & $.003-.01$ & .003 & .001 & 2.95 & .003 & $.001-.005$ \\
Interaction time pre- x post-results & .017 & .01 & 3.35 & $<.001$ & $.01-.03$ & -.001 & .005 & -0.17 & .867 & $-.01-.01$ \\
\hline
\end{tabular}

Note. PA = Positive Affect; NA = Negative Affect. * To evaluate whether time after results release significantly predicted changes in participants' running affect correlations, we tested the same piecewise regression model as described in our statistical analyses, but reverse-coded the dummy variable $(0=$ after results release, $1=$ before results release). 
Table 2. Assessing the role of students' momentary and general attributed importance.

\begin{tabular}{|c|c|c|c|c|c|c|c|c|c|c|}
\hline \multirow[b]{2}{*}{ Predictors } & \multicolumn{5}{|c|}{ Exam-related PA-NA correlation } & \multicolumn{5}{|c|}{ Global PA-NA correlation } \\
\hline & $\beta$ & $\mathrm{SE}$ & $t$ & $p$ & $95 \% \mathrm{CI}$ & $\beta$ & SE & $t$ & $p$ & $95 \% \mathrm{CI}$ \\
\hline \multicolumn{11}{|l|}{ 1. Model within-person association } \\
\hline Intercept & -.067 & .03 & -2.61 & .009 & $-.12--.02$ & -.280 & .03 & -9.85 & $<.001$ & $-.34--.22$ \\
\hline Slope momentary importance & -.001 & .001 & -2.06 & .039 & $-.002--.0001$ & .0001 & .001 & 0.09 & .927 & $-.001-.001$ \\
\hline \multicolumn{11}{|l|}{ 2. Model between-person association } \\
\hline Intercept & -.244 & .04 & -6.04 & $<.001$ & $-.32--.16$ & -.358 & .04 & -8.53 & $<.001$ & $-.44--.28$ \\
\hline Interaction intercept $\mathrm{x}$ general importance & -.091 & .05 & -1.94 & .052 & $-.18-.001$ & -.033 & .05 & -0.68 & .494 & $-.13-.06$ \\
\hline Slope time pre-results & -.012 & .004 & -2.73 & .006 & $-.02--.003$ & .004 & .004 & 0.88 & .380 & $-.005-.01$ \\
\hline Interaction slope time pre-results $\mathrm{x}$ general importance & -.012 & .005 & -2.29 & .022 & $-.02--.002$ & .002 & .005 & 0.52 & .605 & $-.007-.01$ \\
\hline Slope time post-results * & .005 & .001 & 5.36 & $<.001$ & $.003-.007$ & .003 & .001 & 2.94 & .003 & $.001-.005$ \\
\hline Interaction slope time post-results $\mathrm{x}$ general importance $*$ & .0003 & .001 & 0.24 & .808 & $-.002-.003$ & .00002 & .001 & -0.02 & .988 & $-.002-.002$ \\
\hline Interaction time pre- $\mathrm{x}$ post-results & .017 & .005 & 3.39 & $<.001$ & $.007-.03$ & -.001 & .005 & -0.17 & .868 & $-.01-.01$ \\
\hline Interaction time pre- $\mathrm{x}$ post-results $\mathrm{x}$ general importance & .012 & .006 & 2.00 & .045 & $.0003-.02$ & -.003 & .006 & -0.46 & .647 & $-.01-.01$ \\
\hline
\end{tabular}

Note. $\mathrm{PA}=$ Positive Affect; NA = Negative Affect. * To evaluate whether time after results release significantly predicted changes in participants' running affect correlations in the between-person model, we tested the same piecewise regression as described in our statistical analyses, but reverse-coded the dummy variable $(0=$ after results release, $1=$ before results release). Momentary importance scores were within-person centered (meaning that these analyses test the effect of students rating their exam results more or less important than their average), and general importance scores were grand-mean centered. 


\section{Figures}
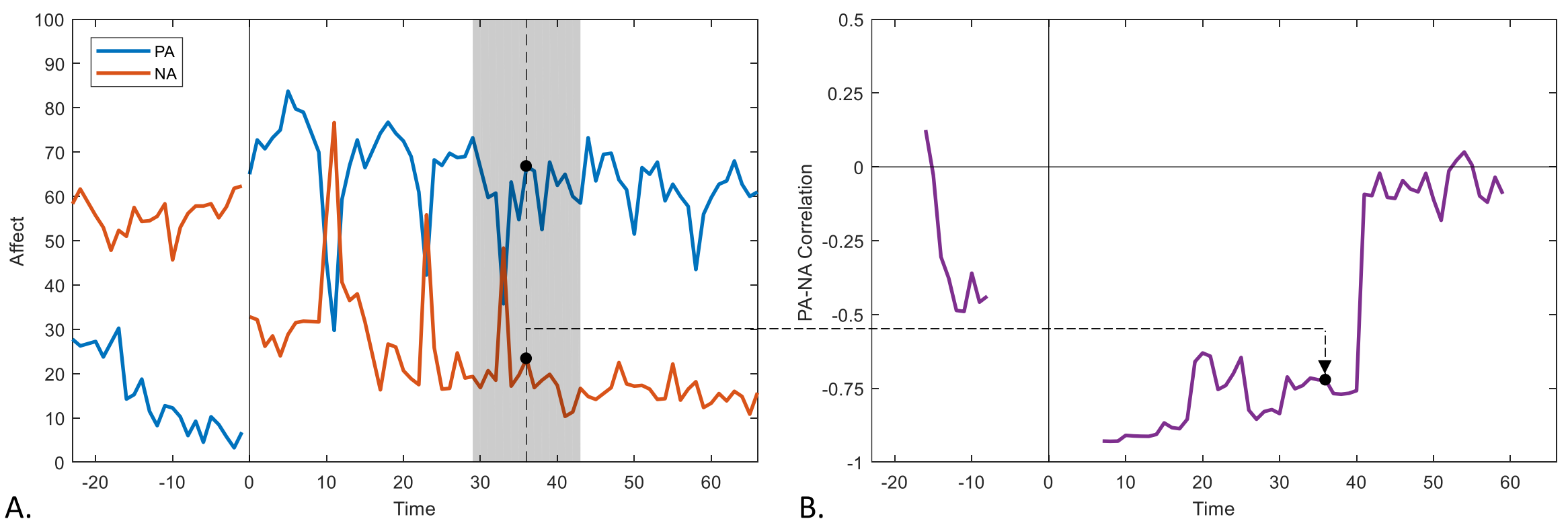

Figure 1. Capturing the running correlation between exam-related PA and NA for one example participant in our dataset. Panel A displays the positive and negative exam-related emotional trajectories before and after exam results release. PA and NA time series were centered around the release of each participants' exam grades, with zero indicating the first measurement occasion after exam results were accessed. The grey band depicts an example sliding window of 15 data points on which exam-related PA-NA running correlations were computed. Panel B visualises the corresponding exam-related PA-NA running correlations calculated from the emotional time series in Panel A. Obtained exam-related PA-NA running correlations were placed at the time point that corresponded with the midpoint of each window. Missing values around the time exam results were released are a result of the fact that running correlations could not be based on both pre- and post-results affective information simultaneously. PA = Positive Affect; NA = Negative Affect. 


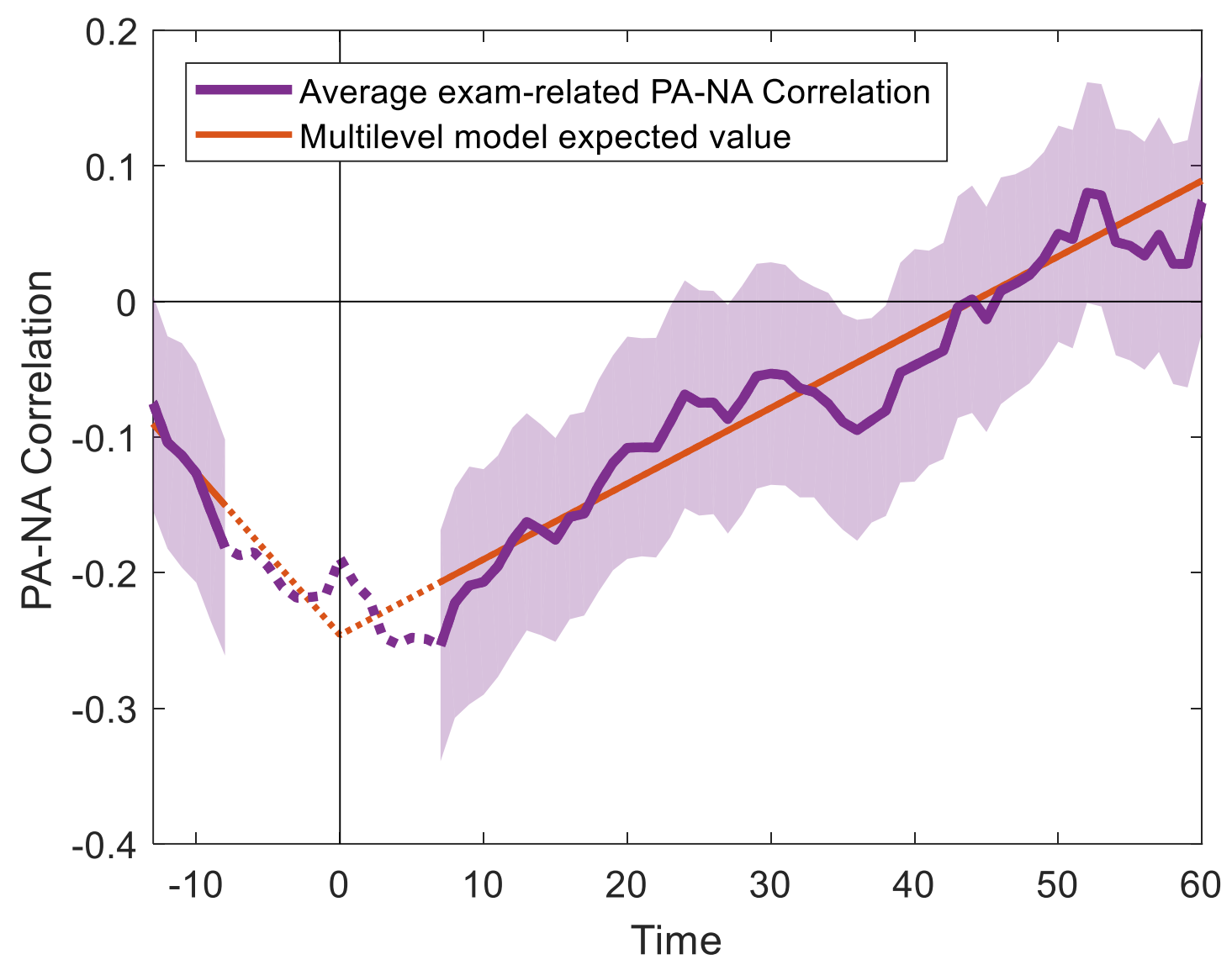

Figure 2. Empirical evidence for a dynamic affective system in response to personally relevant events. The purple line visualises the average exam-related PA-NA correlation observed at a particular moment, with the light purple band depicting its $95 \%$ confidence interval. The zero on the $\mathrm{x}$-axis refers to the first measurement occasion after exam grades were accessed. Missing values around the time exam results were released are a result of the fact that running correlations could not be based on both pre- and postresults affective information simultaneously. The dashed lines indicate how students' exam-related PANA correlation would look if running affect correlations were computed from moving windows that decreased in window size (because they hit the time of the event), but these values were not used to estimate the model. $\mathrm{PA}=$ Positive Affect; NA = Negative Affect. 